$\S=-1$

\title{
Hierarchical Porous Batio 3 Nano-Hexagons as A Visible Light Photocatalyst
}

\author{
Harsha Bantawal and D Krishna Bhat* \\ Department of Chemistry, National Institute of Technology Karnataka, Surathkal, Mangalore-575025, India \\ *Corresponding author E-mail: denthajekb@gmail.com, kishan@nitk.edu.in
}

\begin{abstract}
Hierarchical porous $\mathrm{BaTiO}_{3}$ nano-hexagons was synthesized via a simple hydrothermal route by using $\mathrm{TiO}_{2}$ and $\mathrm{Ba}(\mathrm{OH})_{2} .8 \mathrm{H}_{2} \mathrm{O}$ as starting materials under alkaline environment and its photocatalytic activity was evaluated under visible light by taking methylene blue (MB) as a model pollutant. The prepared $\mathrm{BaTiO}_{3}$ was characterized by powder X-ray diffraction (XRD), X-ray photoelectron spectroscopy (XPS), field emission scanning electron microscopy (FESEM), energy dispersive X-rays analysis (EDX), high resolution transmission electron microscopy (HRTEM), Brunauer-Emmett-Teller (BET) analysis and diffused reflectance spectroscopy (DRS) techniques. It is noteworthy that the $\mathrm{BaTiO}_{3}$ nano-hexagons exhibited significant photocatalytic activity towards the degradation of MB under visible light irradiation. This significant photocatalytic activity of $\mathrm{BaTiO}_{3}$ under visible light is mainly attributed to the special morphology and formation of $\mathrm{Ti}^{3+}$ defects.
\end{abstract}

Keywords: Advanced oxidation process, Barium titanate, Hydrothermal, Perovskite, Photocatalysis, Titanium oxide

\section{Introduction}

Water is imperative to life. The major portion of the water is constituted by aquatic resources such as salted waters of oceans, seas and glaciers. About only $0.65 \%$ of the water total mass can be usable by man. Therefore, there is an imperative need of developing potent and eco-friendly techniques to treat waste waters. Advanced Oxidation Processes (AOPs) have been defined by Glaze et al. ${ }^{1}$ play a vital role in the removal of persistent organic pollutants. Photocatalysis is an integral part of the AOPs has been commonly employed in the removal of persistent organic pollutants because of its zero waste scheme and biocompatibility. ${ }^{2}$ As we know that nearly $43 \%$ of the solar irradiation energy corresponds to the visible light and nearly $7 \%$ of the energy corresponds to the UV light. Undeniably, the first generation $\mathrm{TiO}_{2}$ is a prominent photocatalyst in the UV region, due to its wide band gap of $3.2 \mathrm{eV}$ hamper its use in visible range. Therefore, there is an imperative need in extension of visible light response of wide band gap photocatalysts.

Perovskite alkaline earth titanates $\left(\mathrm{ATiO}_{3}, \mathrm{~A}=\mathrm{Ca}, \mathrm{Ba}, \mathrm{Sr}\right)$ are found to exhibit compelling properties in various applications like photocatalytic degradation of organics, photocatalytic water splitting and photo reduction of carbon dioxide due to their wide band gap, excellent catalytic activity, excellent chemical stability and good biocompatibility. ${ }^{3}$ Barium titanate $\left(\mathrm{BaTiO}_{3}\right)$, a ternary metal titanate perovskite with a high dielectric constant and ferroelectric properties has been extensively investigated. It is also a very important semiconductor under UV light due to its band gap of 3.25 $\mathrm{eV}$ and its photocatalytic activity is hampered in visible light. Therefore, several probes have been explored in order to enhance the photocatalytic activity of $\mathrm{BaTiO}_{3}$ such as surface sensitization, ion doping, noble metal loading and heterostructural strategy. On the other side morphology has an imperative impact on photocata- lytic activity. Yingpu et al. ${ }^{4}$ studied the facet effect of $\mathrm{Ag}_{3} \mathrm{PO}_{4}$ sub-microcrystals on photocatalytic properties. They found that the morphology control of semiconductors enables selective exposure of photocatalytically active facets, which might be applicable in the design of highly efficient visible light active catalysts. Yan et. al. ${ }^{5}$ synthesized rod like $\mathrm{CaTiO}_{3}$. They found that the band gap of $\mathrm{CaTiO}_{3}$ with this special morphology was narrowed.

Hydrothermal method has been employed for the fabrication of perovskite oxide owing to its high purity, homogeneity, crystallinity, well defined morphology and controllable particle size. ${ }^{6}$ To the best of our knowledge there has been no work devoted to the photocatalytic performance of $\mathrm{BaTiO}_{3}$ nano-hexagons under visible light irradiation. In this work, we have synthesized $\mathrm{BaTiO}_{3}$ hydrothermally using $\mathrm{TiO}_{2}, \mathrm{Ba}(\mathrm{OH})_{2} .8 \mathrm{H}_{2} \mathrm{O}$ and $\mathrm{NaOH}$ as raw materials and the as synthesized sample was thoroughly characterized by XRD, FESEM, TEM, HRTEM, BET, XPS and UV-visible diffuse reflectance spectroscopic techniques. The as prepared $\mathrm{BaTiO}_{3}$ showed narrowed band gap owing to its special morphology and $\mathrm{Ti}^{3+}$ defects. The photocatalytic activity of the as synthesized sample was evaluated by taking methylene blue (MB) as the target pollutant under visible light irradiation.

\section{Materials and method}

Titanium(IV) oxide, anatase, 99.6\% (metal basis)-325 Mesh Powder was procured from Alfa Aesar, Barium Hydroxide Extra pure (octahydrate) was procured from Loba Chemie Pvt. Ltd. and $\mathrm{NaOH}$ pellets were procured from Finar limited. All the chemicals were used without further purification. All the reactions were carried out with Millipore water.

$\mathrm{BaTiO}_{3}$ nanopowder was synthesized in a beaker by taking $0.5 \mathrm{~g}$ Titanium(IV) oxide powder, $1.75 \mathrm{~g}$ Barium Hydroxide (octahydrate) and $50 \mathrm{~mL}$ of $5 \mathrm{M} \mathrm{NaOH}$. The mixture was magnetically stirred for 20 minutes and then placed in an ultrasonic bath for 5 
minutes. The resultant mixture was transferred to a $100 \mathrm{~mL}$ Teflon lined autoclave and heated to $120^{\circ} \mathrm{C}$ for 48 hours. The precipitated powders were cooled to room temperature and neutralized to $\mathrm{pH} 7$ with the help of $5 \mathrm{M} \mathrm{HCl}$ solution. Excess liquid was removed by decantation and the white precipitate obtained was recovered by centrifugation with water. Finally, the obtained solid was dried at $80^{\circ} \mathrm{C}$ overnight.

The structural characterization of the synthesized material was performed using an X-ray diffractometer (XRD, Rigaku) with monochromatic $\mathrm{Cu}-\mathrm{K} \alpha$ radiation $(\lambda=0.154 \mathrm{~nm})$ at a scan rate of $2^{\circ}$ per minute in the range of $5-80^{\circ}$. The surface morphology was observed using FESEM (Carl Zeiss), TEM (Fie Tecnai), HRTEM (Fie Tecnai). The elemental analysis of the synthesized material was performed by using EDX (Carl Zeiss) and X-ray photoelectron spectrometer (Kratos XSAM800) equipped with a standard monochromatic $\mathrm{Al} \mathrm{K} \alpha$ source. The specific surface area was determined using Brunauer-Emmett-Teller (BET) method (BEL SORP II, JAPAN) based on adsorption data in the relative pressure in the range of $0.05-0.03$. The pore size distribution was determined using the Barrett-Joyner-Halenda (BJH) method applied to the desorption branch. Diffuse reflectance spectrum was recorded using UV-Visible diffuse reflectance spectrometer (DRS, Analytic Jena).

The photocatalytic activity of the synthesized material was evaluated by taking methylene blue (MB) solution using a photocatalytic reactor under ambient atmospheric conditions. The photocatalytic reactor was equipped with a high - pressure $250 \mathrm{~W} \mathrm{Hg}$ vapour lamp operating at a wavelength of $410-700 \mathrm{~nm}$ was used as a source of visible light. In these experiments $100 \mathrm{~mL}$ of MB solution $\left(5.5 \mathrm{mg} \mathrm{L}^{-1}\right)$ and photocatalyst $(50 \mathrm{mg})$ were taken in to a 500 $\mathrm{mL}$ of pyrex glass beaker and magnetically stirred for 30 minutes in order to reach adsorption-desorption equilibrium of the catalyst. Then the solution was irradiated with visible light radiation. During photocatalytic studies, at regular intervals, $5 \mathrm{~mL}$ of the reacted MB solution was sampled out, centrifuged and the absorbance of the supernatant dye solution was measured using UV - visible spectrometer at $664 \mathrm{~nm}$. The percentage of degradation of the dye was calculated as per equation (1). ${ }^{7}$

Degradation $\%=\left[\left(\mathrm{A}_{0}-\mathrm{A}_{\mathrm{t}}\right) / \mathrm{A}_{0}\right] \times 100$

Where, $A_{0}$ is the initial absorbance of dye solution and $A_{t}$ is the absorbance at different intervals of time.

\section{Results and Discussions}

\subsection{XRD Studies}

Figure 1 shows the XRD patterns of the as prepared $\mathrm{BaTiO}_{3}$. The diffraction peaks at $22.01^{\circ}, 31.40^{\circ}, 38.76^{\circ}, 45.01^{\circ}, 50.65^{\circ}, 55.96^{\circ}$ and $65.63^{\circ}$ were ascribed to the (100), (110), (111), (200), (210), (211) and (220) crystal planes of $\mathrm{BaTiO}_{3}$ and confirm well to JCPDS file no. 01-075-0461. The average crystal size of $\mathrm{BaTiO}_{3}$ was found to be $40.74 \mathrm{~nm}$ which was calculated from Scherrer equation. $^{8}$

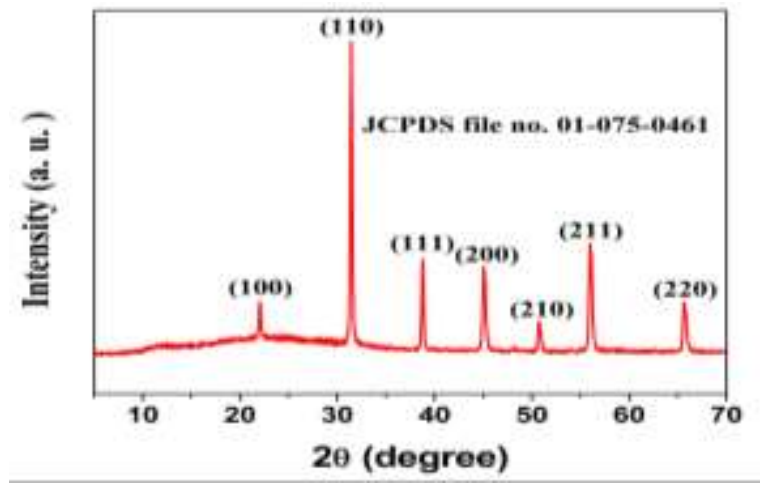

Fig. 1: XRD spectrum of $\mathrm{BaTiO}_{3}$

\subsection{BET Surface Area Analysis}

(a)

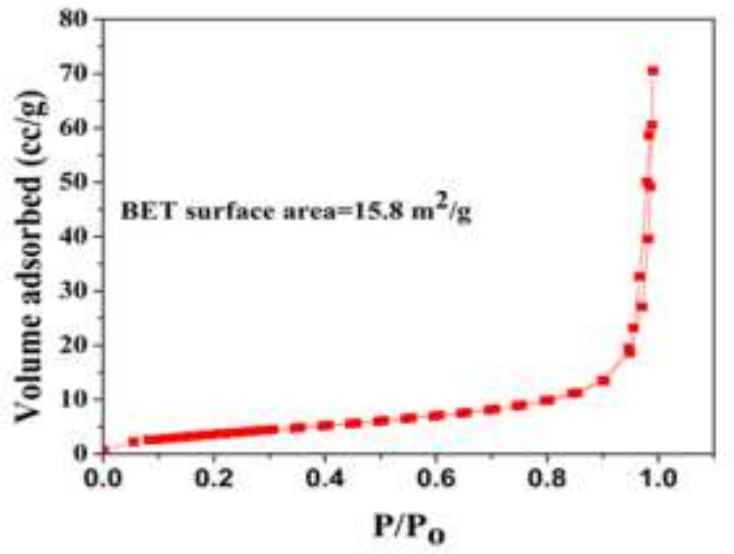

(b)

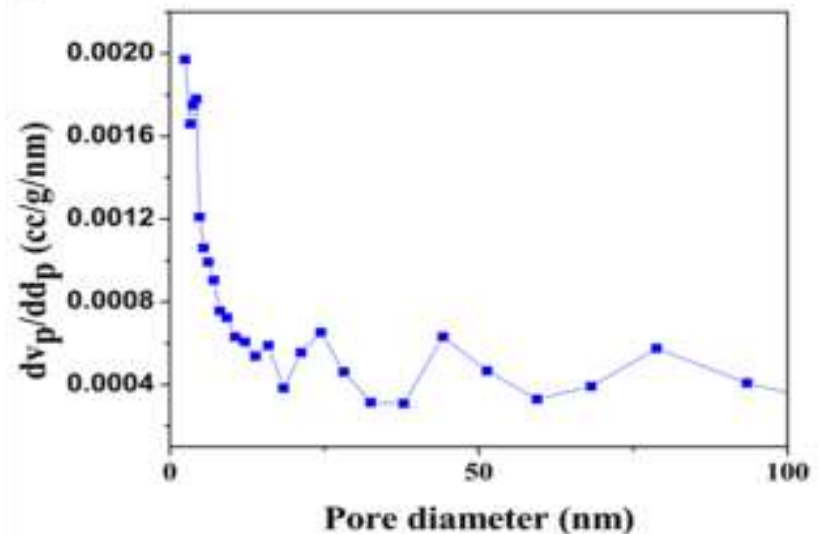

Fig. 2: BET surface area analysis: Adsorption-desorption isotherms

The nitrogen adsorption-desorption isotherms were used to obtain information about the BET specific surface area and pore size distribution in the synthesized $\mathrm{BaTiO}_{3}$. The nitrogen adsorptiondesorption isotherm of $\mathrm{BaTiO}_{3}$ exhibited hysteresis loop at relative pressure $\left(\mathrm{P} / \mathrm{P}_{\mathrm{o}}\right)$ close to unity is shown in Figure 2 (a), indicating the presence of large mesopores and macropores, which can be assorted as type IV according to IUPAC classification. This isotherm exhibited $\mathrm{H} 3$ hysteresis loop associated with slit like pores. ${ }^{9}$ BET analysis showed that the specific surface area of $\mathrm{BaTiO}_{3}$ is $15.86 \mathrm{~m}^{2} \mathrm{~g}^{-1}$. The BJH pore size distribution is broad in the range of 2-100 nm, indicating the presence of mesopores and macropores is shown in Figure 2 (b). This hierarchical meso/macro porous $\mathrm{BaTiO}_{3}$ nano-hexagons is believed to enhance the photocatalytic activity due to the increasing photo-absorption efficiency and efficient diffusion of molecules caused by the macropores. ${ }^{10}$

\subsection{Morphology Studies}

The morphology of the synthesized $\mathrm{BaTiO}_{3}$ was studied with the help of electron microscopy. The FESEM images of the $\mathrm{BaTiO}_{3}$ suggest that the particles have hexagon shape is shown in Figure 3 (a) with red circles. The EDX analysis was carried out for the synthesized $\mathrm{BaTiO}_{3}$. The EDX spectrum confirms the presence of barium, titanium and oxygen elements is shown in Figure 3 (b). Figure 3 (c) shows the HRTEM image of $\mathrm{BaTiO}_{3}$. The lattice fringe of about $0.143 \mathrm{~nm}$ agrees well with the (220) plane of cubic $\mathrm{BaTiO}_{3}$ structure. The bright spots of SAED pattern further shows that $\mathrm{BaTiO}_{3}$ is of single crystalline is shown Figure 3 (d). 

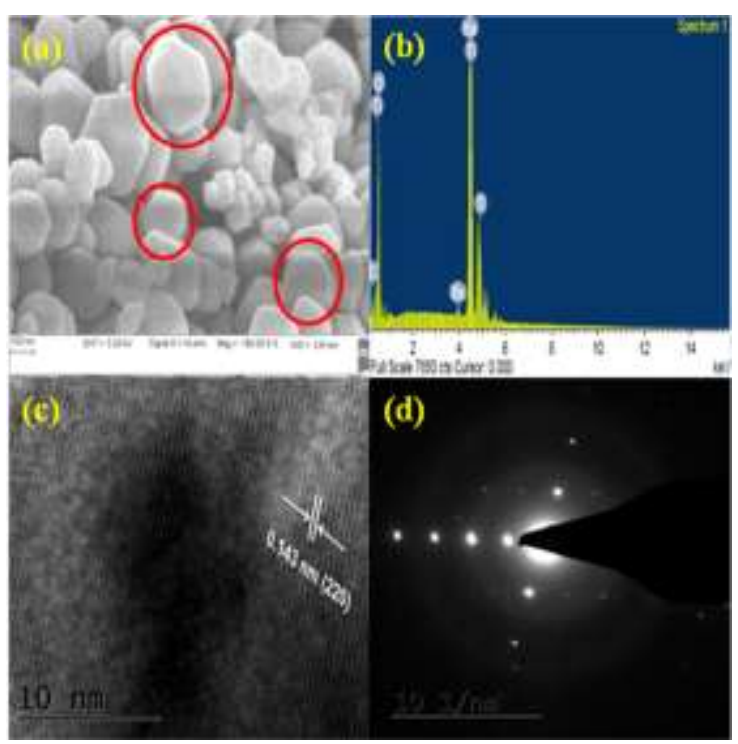

Fig. 3: (a) FESEM image of $\mathrm{BaTiO}_{3}$ nano-hexagons, (b) EDX spectrum of BaTiO3, (c) HRTEM image of $\mathrm{BaTiO}_{3}$, (d) SAED pattern of $\mathrm{BaTiO}_{3}$

\subsection{XPS Analysis}

X-ray photoelectron spectroscopy (XPS) measurement was carried out in order to determine the oxidation state and electronic environment of the elements. Figure 4 (a) shows the high-resolution $\mathrm{Ba} 3 \mathrm{~d}$ XPS spectrum of $\mathrm{BaTiO}_{3}$. The two peaks at $793.65 \mathrm{eV}$ and $778.35 \mathrm{eV}$ could be assigned to the splitting of the $\mathrm{Ba} 3 \mathrm{~d}_{3 / 2}$ and $\mathrm{Ba} \mathrm{d}_{5 / 2}$ spin states respectively. Figure 4 (c) shows the narrow scan O 1s XPS spectrum. The two peaks at $529.25 \mathrm{eV}$ and 530.95 $\mathrm{eV}$ were assigned to the lattice oxygen (Ti-O bond) and hydroxyl group or absorbed oxygen in the as synthesized $\mathrm{BaTiO}_{3}$. Figure 4 (b) shows the narrow scan spectra Ti $2 \mathrm{p}$ XPS spectrum. The two peaks at $457.95 \mathrm{eV}$ and $463.45 \mathrm{eV}$ could be ascribed to the splitting of the Ti $2 p_{3 / 2}$ and Ti $2 p_{1 / 2}$ spin states respectively. ${ }^{11}$ The binding energy of Ti $2 \mathrm{p}_{3 / 2}$ is found to be $457.95 \mathrm{eV}$, which is blue shifted as compared to the reported values for $\mathrm{BaTiO}_{3}$ bulks. This downshift in the binding energy is mainly ascribed to the formation of $\mathrm{Ti}^{3+}$ defects. Some of the $\mathrm{Ti}^{4+}$ in $\mathrm{BaTiO}_{3}$ was transformed to $\mathrm{Ti}^{3+}$ in order to balance the oxygen vacancies. ${ }^{12}$ the existence of these defects was found to boost the absorption of light in the visible region by upshifting the valence band edge. ${ }^{13}$

(a)

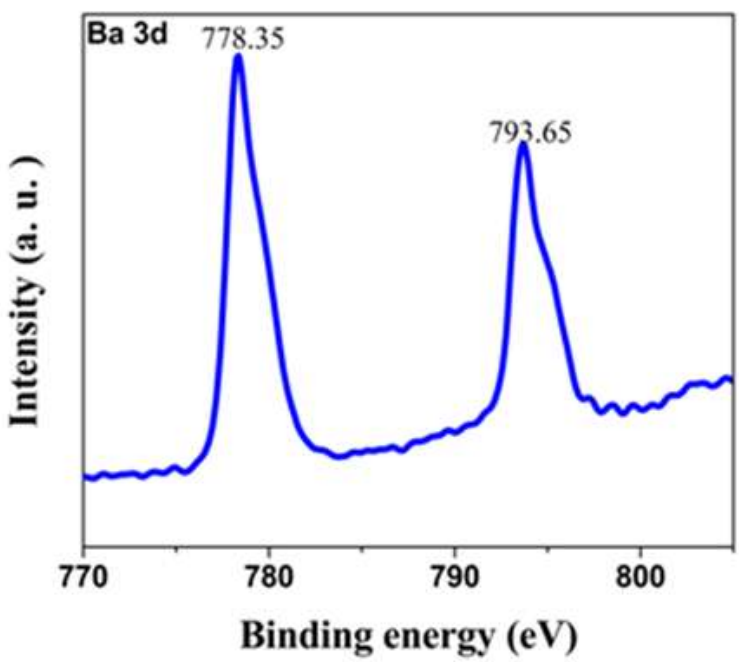

(b)

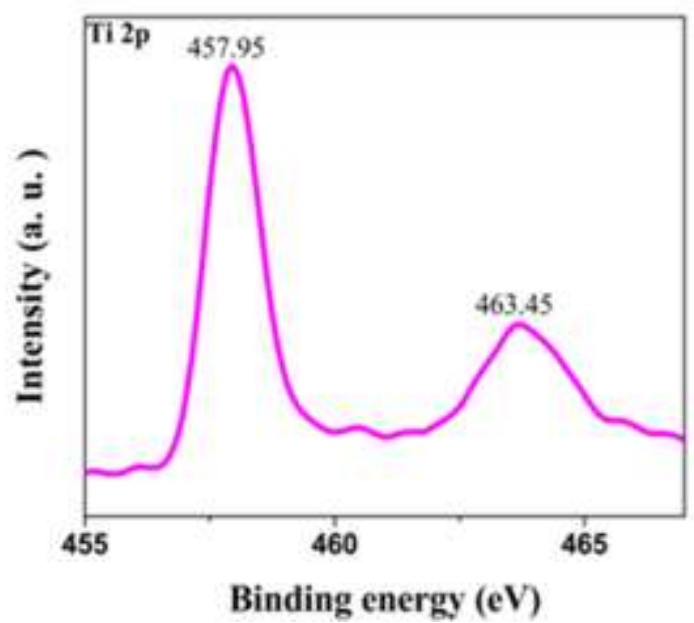

(c)

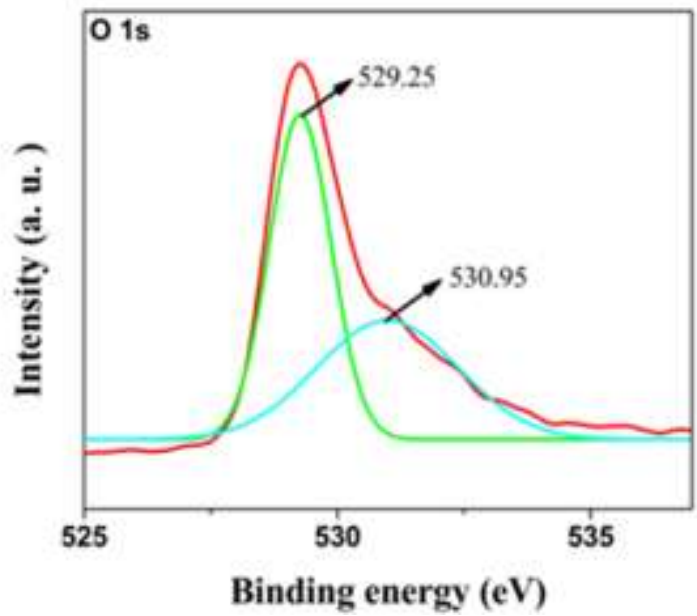

Fig. 4: (a) XPS spectrum of $\mathrm{Ba} 3 \mathrm{~d}$ peaks in $\mathrm{BaTiO}_{3}$, (b)XPS spectrum of Ti 2p peaks in $\mathrm{BaTiO}_{3}$, (c)XPS spectrum of $\mathrm{O}$ 1s peaks in $\mathrm{BaTiO}_{3}$

\subsection{Optical Absorbance Analysis}

The band gap is the most crucial factor with respect to the photocatalytic activity of the materials. Figure 5 shows the UV - visible DRS of $\mathrm{BaTiO}_{3}$. It can be seen that the absorption edge of $\mathrm{BaTiO}_{3}$ was found to be ca. $426.63 \mathrm{~nm}$. The $\mathrm{BaTiO}_{3}$ band gap energy was calculated to be $2.90 \mathrm{eV}$ using the relation $\mathrm{E}_{\mathrm{g}}=1240 / \lambda$, where $\lambda$ is the onset absorption wavelength ${ }^{14}$ This result clearly suggest that the $\mathrm{BaTiO}_{3}$ can perform efficiently as a photocatalyst under visible light.

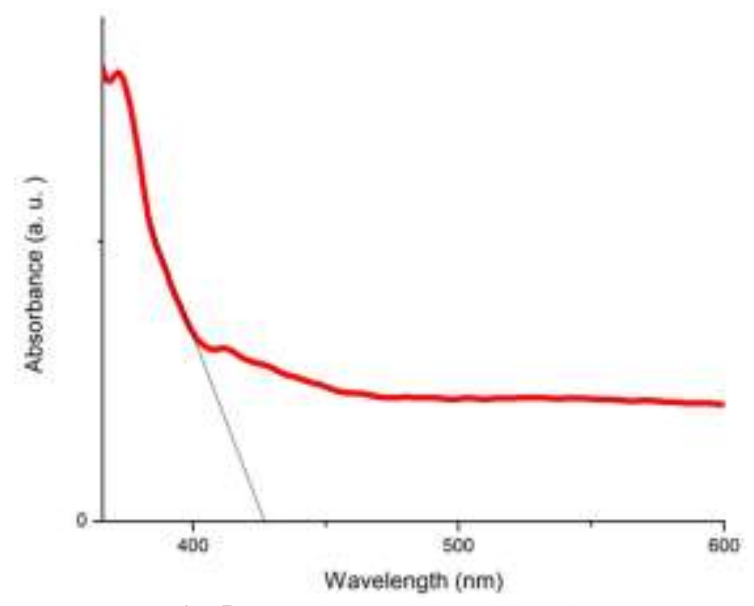

Fig. 5: UV-Vis DRS spectra of $\mathrm{BaTiO}_{3}$ 


\subsection{Photocatalytic Activity}

The photocatalytic activity of $\mathrm{BaTiO}_{3}$ was evaluated under visible light irradiation by taking methylene blue as a target pollutant. The mixture of $\mathrm{MB}$ solution and $\mathrm{BaTiO}_{3}$ (photocatalyst) was magnetically stirred for 30 minutes in the dark in order to reach adsorption-desorption of the photocatalyst. A blank test was carried out in the absence of photocatalyst, there was no significant degradation. Figure 6 shows the photocatalytic degradation of $\mathrm{BaTiO}_{3}$. The percentage degradation was found to be $92.8 \%$.

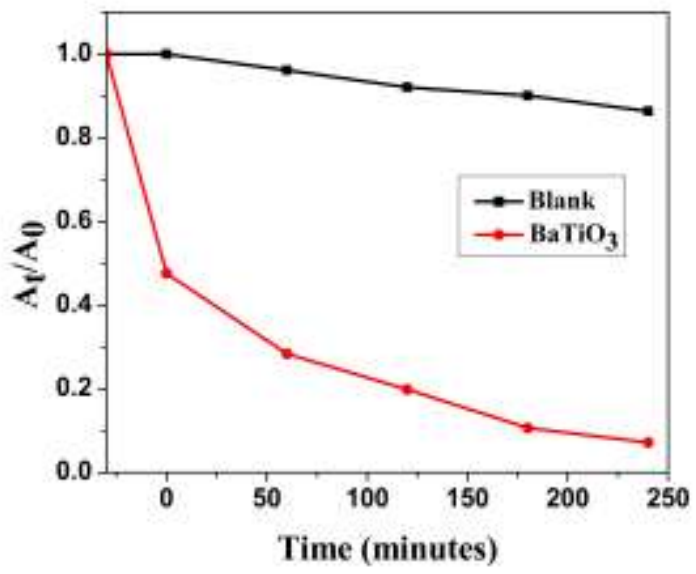

Fig. 6: Degradation rate of $\mathrm{MB}$ by $\mathrm{BaTiO}_{3}$

The photocatalytic degradation of methylene blue by the synthesized $\mathrm{BaTiO}_{3}$ nano-hexagons followed a pseudo-first order kinetic equation ${ }^{15}$ as given in equation (2) is shown in figure 7 .

$\ln \left(\mathrm{C}_{\mathrm{t}} / \mathrm{C}_{0}\right)=-\mathrm{kt}$

Where, $\mathrm{C}_{0}$ is the initial concentration, $\mathrm{C}_{\mathrm{t}}$ is the concentration at irradiation time $(\mathrm{t})$ and $\mathrm{k}$ is the first order rate constant. The rate constant value was measured from the slope of the straight line. The rate constant value was found to be 0.0091

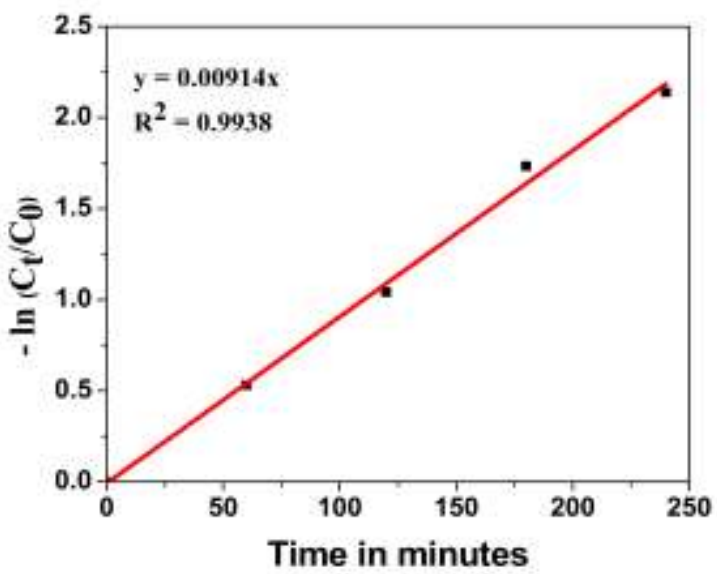

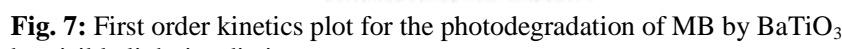
by visible light irradiation

The reusability of the photocatalysts is another deciding factor for their photocatalytic applications. The stability of the $\mathrm{BaTiO}_{3}$ nanohexagons was tested by recycling the photocatalytic experiment. It was found that there is a little loss in the photocatalytic degradation efficiency even after five cycles is shown in figure 8 . This indicates that the $\mathrm{BaTiO}_{3}$ nano-hexagons possess enough stability towards photocatalytic degradation reactions.

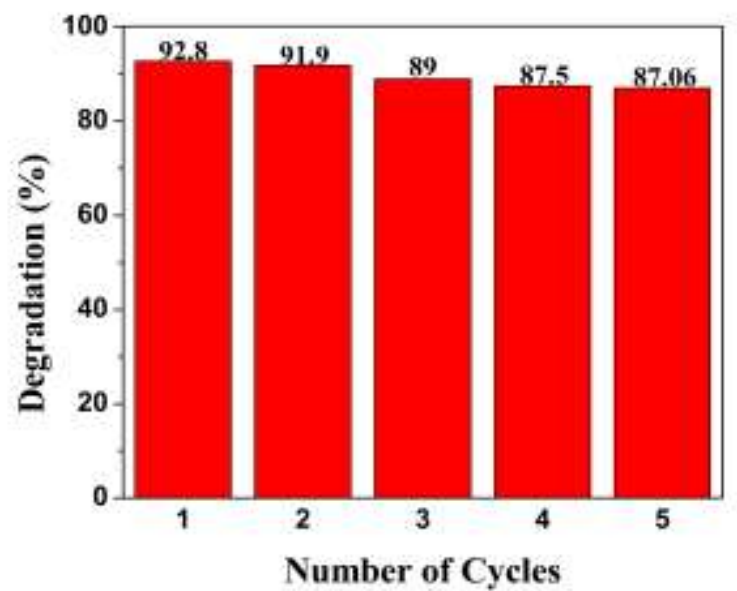

Fig. 8: Recyclability of $\mathrm{BaTiO}_{3}$ for the degradation of $\mathrm{MB}$ under visible light irradiation

\subsection{Mechanism of Photocatalytic Activity}

The enhanced photocatalytic activity of the $\mathrm{BaTiO}_{3}$ can be mainly attributed to the efficient photo absorption in the visible region and the efficient generation, separation and migration of the photoinduced charges to the surface of $\mathrm{BaTiO}_{3}$. The valance band (VB) edge position and the conduction band (CB) position of the $\mathrm{BaTiO}_{3}$ were calculated with the help of the Mulliken electronegativity theory ${ }^{16}$ following eqn. (3) and (4)

$E_{V B}=\chi-E^{e}+0.5 E_{g}$

$\mathrm{E}_{\mathrm{CB}}=\mathrm{E}_{\mathrm{VB}}-\mathrm{E}_{\mathrm{g}}$

Where $\mathrm{E}_{\mathrm{VB}}$ is the VB edge potential, $\mathrm{E}_{\mathrm{CB}}$ is the $\mathrm{CB}$ edge potential, $\mathrm{E}_{\mathrm{g}}$ is the energy band gap of the semiconductor, $\chi$ is the absolute electronegativity of the semiconductor which is calculated as the geometric mean of the electronegativity of the constituent atoms and $E^{\mathrm{e}}$ is the energy of free electrons on the hydrogen scale (-4.5 eV). $\mathrm{E}_{\mathrm{VB}}$ and $\mathrm{E}_{\mathrm{CB}}$ of $\mathrm{BaTiO}_{3}$ were calculated and listed in Table 1.

Table 1. $\mathrm{E}_{\mathrm{VB}}$ and $\mathrm{E}_{\mathrm{CB}}$ of $\mathrm{BaTiO}_{3}$

\begin{tabular}{c|c|c|c|c|} 
Sample & $\chi(\mathrm{eV})$ & $\mathrm{E}_{\mathrm{g}}(\mathrm{eV})$ & $\mathrm{E}_{\mathrm{VB}}(\mathrm{eV})$ & $\mathrm{E}_{\mathrm{CB}}(\mathrm{eV})$ \\
$\mathrm{BaTiO}_{3}$ & 5.27 & 2.90 & 2.22 & -0.68 \\
\hline
\end{tabular}

\subsection{Degradation Mechanism}

When the $\mathrm{BaTiO}_{3}$ nano-hexagons are irradiated with a visible light of energy equal to $2.90 \mathrm{eV}$, electrons present in the valence band move to the newly formed $\mathrm{Ti}^{3+}$ state. As shown in figure 9 , the generated electrons probably reacted with dissolved oxygen molecules to form superoxide radical anions $\left(\mathrm{O}_{2}^{-}\right)$, which on protonation resulted in the formation of hydroperoxy radical $\left(\mathrm{OOH}^{*}\right)$, which finally resulted in the formation of hydroxyl radicals $\left(\mathrm{OH}^{*}\right)$. On the other hand, the generated holes will react with $\mathrm{MB}$ or react with surface adsorbed water to generate hydroxyl radicals. The formed hydroxyl radical was a strong oxidizing agent to decompose the organic dyes to harmless products.

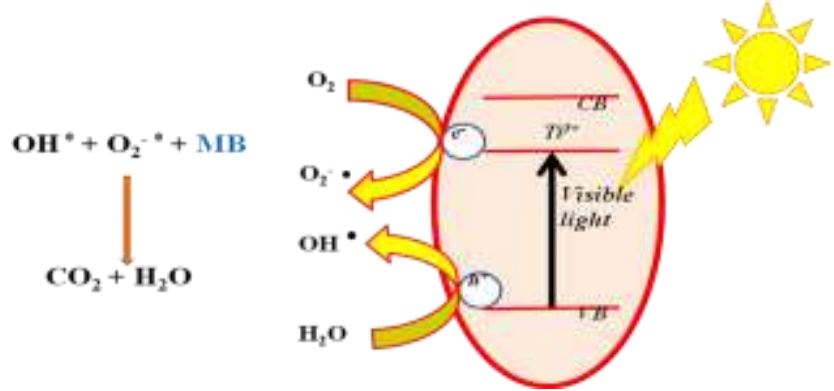

Fig. 9:. Mechanism of photodegradation of $\mathrm{MB}$ by $\mathrm{BaTiO}_{3}$ under visible light irradiation 
The improvement in the photocatalytic activity of $\mathrm{BaTiO}_{3}$ under visible light might be due to the special morphology of $\mathrm{BaTiO}_{3}$ as well as formed $\mathrm{Ti}^{3+}$ defects. The morphology control of semiconductors enables selective exposure of photocatalytically active facets, which might be applicable in the design of highly efficient visible light active catalysts. The existence of $\mathrm{Ti}^{3+}$ defects or oxygen vacancies was found to boost the absorption of light in the visible region by upshifting the valance band edge. However, an ideal concentration of oxygen vacancies is required to enhance the photocatalytic activity.

\section{Conclusion}

Hierarchical porous $\mathrm{BaTiO}_{3}$ nano-hexagons was synthesized successfully by one pot simple hydrothermal route by using $\mathrm{TiO}_{2}$ and $\mathrm{Ba}(\mathrm{OH})_{2} .8 \mathrm{H}_{2} \mathrm{O}$ as starting materials under alkaline environment and its photocatalytic activity was evaluated under visible light by taking methylene blue (MB) as a target pollutant. The structural, surface morphological and optical properties of the as prepared $\mathrm{BaTiO}_{3}$ was characterized by powder X-ray diffraction (XRD), X-ray photoelectron spectroscopy (XPS), field emission scanning electron microscopy (FESEM), energy dispersive X-rays analysis (EDX), high resolution transmission electron microscopy (HRTEM), Brunauer-Emmett-Teller (BET) analysis and diffused reflectance spectroscopic (DRS) techniques. It is noted that the $\mathrm{BaTiO}_{3}$ nano-hexagons exhibited significant photocatalytic activity towards the degradation of $\mathrm{MB}$ under visible light irradiation. This significant photocatalytic activity of $\mathrm{BaTiO}_{3}$ under visible light is mainly attributed to the special morphology and formation of $\mathrm{Ti}^{3+}$ defects. The $\mathrm{BaTiO}_{3}$ showed high activity as well as high stability even after 5 cycles of photocatalytic processes. The observed results confirmed that the synthesized $\mathrm{BaTiO}_{3}$ nanohexagons can be a potential and eco-friendly photocatalyst under visible light.

\section{Acknowledgement}

HB is thankful to the National Institute of Technology Surathkal for providing research facilities and financial assistance in the form of an institute fellowship.

\section{References}

[1] R. Vinu \& G Madras (2010), Environmental remediation by photocata lysis. Journal of the Indian Institute of Science 90, 189-230.

[2] Y. Wang, C. G. Niu, L. Wang, Y. Wang, X. G. Zhang \& G. M Zheng (2016), Synthesis of fern-like $\mathrm{Ag} / \mathrm{AgCl} / \mathrm{CaTiO} 3$ plasmonic photocatalysts and their enhanced visible-light photocatalytic properties. RSC Advances 6, 47873-47882.

[3] C. K. Chen, S. X. Zhao, Q. L. Lu, K. Luo, X. H. Zhang \& C. W. Nan (2017), Topochemical synthesis and photocatalytic activity of 3D hierarchical $\mathrm{BaTiO} 3$ microspheres constructed from crystalaxis-oriented nanosheets. Dalton Transactions 46, 5017-5024.

[4] Y. Bi, S. Ouyang, N. Umezava, J. Cao \& J. Ye (2011), Facet Effect of Single-Crystalline Ag3PO4 Sub-microcrystals on Photocatalytic Properties. Journal of American Chemical Society 133, 6490-6492.

[5] X. Yan, X. Huang, Y. Fang, Y. Min, Z. Wu, W. Li, J. Yuan \& L. Tan (2014), Synthesis of Rodlike CaTiO3 with Enhanced Charge Separation Efficiency and High Photocatalytic Activity. International Journal of Electrochemical Science 9, 5155-5163.

[6] X. Xiong, R. Tian, X. Lin, D. Chu \& S. Li (2015), Formation and Photocatalytic Activity of BaTiO3Nanocubes via Hydrothermal Process. Journal of Nanomaterials 2015, 1-6.

[7] P. Saikia, A. T. Miah \& P. P. Das (2017), Highly efficient catalytic reductive dehydration of various organic dyes by $\mathrm{Au} / \mathrm{Ce}-\mathrm{TiO} 2$ nano-hybrid. Journal of Chemical Sciences 129, 81-93.

[8] M. M. J. Sadiq, U. S. Shenoy \& D. K. Bhat (2016), Novel RGOZnWO4-Fe3O4 nanocomposite as high performance visible light photocatalyst. RSC Advances 6, 61821-61829.
[9] Q. Xiang, J. Yu \& M. Jaroniec (2011), Enhanced photocatalytic $\mathrm{H} 2$ - production activity of graphene-modified titania nanosheets. Nanoscale 3, 3670-3678.

[10] Y. Kobayashi, T. Nozaki, R. Kanasaki, R. Shoji \& K. Sato (2017), Fabrication of Macroporous TiO2 Loaded with Magnetite for Photocatalytic Degradation of Methylene Blue. Journal of Chemical Engineering Japan 50, 132-135.

[11] W. W. Lee, W. H. Chung, W. S. Huang, W. C. Lin, W. Y. Lin, Y. R. Jiang \& C. C. Chen (2013), Photocatalytic activity and mechanism of nano-cubic barium titanate prepared by a hydrothermal method. Journal of Taiwan Institute of Chemical Engineers 44, 660-669.

[12] Y. Yang, X. Wang, C. Sun \& L. Li (2009), Structure study of single crystal $\mathrm{BaTiO} 3$ nanotube arrays produced by the hydrothermal method. Nanotechnology 20, 1-5.

[13] M. Liu, X. Qiu, M. Miyauchi \& K. Hashimoto (2011), Cu(II) Oxide Amorphous Nanoclusters Grafted Ti3+ Self-Doped TiO2: An Efficient Visible Light Photocatalyst. Chemistry of Materials 23, 52825286.

[14] L. Zhang, Y. Li, Q. Zhang \& H. Wang (2013), Hierarchical nanostructure of WO3 nanorods on TiO2nanofibers and the enhanced visible light photocatalytic activity for degradation of organic pollutants. CrystEngComm, 15, 5986-5993.

[15] M. M. J. Sadiq, U. S. Shenoy \& D. K. Bhat (2017), NiWO4-ZnONRGO ternary nanocomposite as an efficient photocatalyst for degradation of methylene blue and reduction of 4-nitro phenol. Journal of Physics and Chemistry of Solids 109, 124-133.

[16] M. M. J. Sadiq, U. S. Shenoy \& D. K. Bhat (2017), Enhanced photocatalytic performance of $\mathrm{N}$-doped $\mathrm{RGO}-\mathrm{FeWO} / \mathrm{Fe} 3 \mathrm{O} 4$ ternary nanocomposite in environmental applications. Materials Today Chemistry 4, 133-141. 